\title{
Gluteus Medius
}

National Cancer Institute

\section{Source}

National Cancer Institute. Gluteus Medius. NCI Thesaurus. Code C52933.

One of the three gluteal muscles on the lateral surface of the pelvic bone that abducts and rotates the thigh. 